\title{
Wenyang Huazhuo Tongluo formula, a Chinese herbal decoction, improves skin fibrosis by promoting apoptosis and inhibiting proliferation through down- regulation of survivin and cyclin D1 in systemic sclerosis
}

\author{
Li Han ${ }^{1}$, Hua Bian ${ }^{1 *}$, Jingfeng Ouyang ${ }^{2}$, Yuefeng Bi ${ }^{3}$, Lei Yang ${ }^{1}$ and Songshan Ye
}

\begin{abstract}
Background: Fibrosis is a major contributor to systemic sclerosis (SSC)-related morbidity, and rapid, progressive skin involvement predicts later mortality. Western medicine therapies for SSc cannot produce satisfactory effects currently, while Traditional Chinese Medicine (TCM), such as the Wenyang Huazhuo Tongluo (WYHZTL) formula, a Chinese herbal decoction, has shown amazing anti-fibrosis efficacy on SSc in clinical applications. This study is aiming to investigate the anti-fibrotic mechanism of WYHZTL formula for the treatment of SSc.

Methods: Fibroblasts from primary culture of skin lesions of SSC patients were exposed to rat medicated sera containing WYHZTL or XAV939, a small-molecule inhibitor of both tankyrase 1/2 and Wnt/B-catenin pathway. Cell counting kit-8 assay and Annexin V FITC/PI apoptosis kit were used to analyze cell proliferation and apoptosis in fibroblasts, respectively. Reverse transcription-polymerase chain reaction (RT-PCR) and western blotting were used to detect the mRNA and protein levels of cyclin D1 and survivin.

Results: After 28,48 and $72 \mathrm{~h}$ of incubation, the proliferative ability of the fibroblasts cells was obviously reduced by the sera containing WYHZTL compared with that in the control group; the percentage of apoptotic cell population in the sera containing WYHZTL treated fibroblasts cells was significantly higher than that in those treated with the control sera, and was about similar to that in those treated with XAV939. The sera containing WYHZTL could down-regulate both mRNA and protein levels of cyclin D1 and survivin, compared with the control group.
\end{abstract}

Conclusions: The present study demonstrates the antiproliferative and pro-apoptotic actions of WYHZTL formula against fibroblasts and the effect may be related to the down-regulation of mRNA and protein levels of cyclin D1 and survivin in SSC.

Keywords: Wenyang Huazhuo Tongluo formula, Systemic sclerosis, Anti-fibrosis, Survivin, Cyclin D1

\footnotetext{
* Correspondence: biancrown@163.com

'Zhang Zhongjing College of Chinese Medicine, Nanyang Institute of

Technology, Changjiang Road 80, Nanyang 473004Henan, China

Full list of author information is available at the end of the article
} 


\section{Background}

Systemic sclerosis (SSc) is an autoimmune and/or autoinflammatory disease mainly characterized by the excessive accumulation of extracellular matrix (ECM), especially as collagen, and by extensive fibrosis in skin and eventually in multiple internal organs, such as lung, heart, esophagus and kidney [1]. Genetic factors, infection and unknown environmental influences may play roles in the pathogen of the disease. However, the exact initiating events that lead to the disease remain unclear. Immune dysfunction, vascular damage, abnormal metabolism of connective tissue and a complex interaction of them are thought to be involved in SSc. Fibrosis is a major contributor to the disease related morbidity, and rapid, progressive skin involvement predicts later mortality [2]. Western medicine therapies mainly include immunosuppressive agents, vasoactive drugs and connective tissue formation inhibitors. However, the clinical effects of these therapies are of questionable significance currently, such as methotrexate and cyclophosphamide $[3,4]$.

Considering the unsatisfactory effect of current therapies for SSc, it would be desirable to have novel drugs/ therapies. Traditional Chinese medicine (TCM) has a long history of dealing with diseases and evolved over the past two and a half millennia to become the second largest health-care system in the world, after modern Western medicine. In the past few years, much research has focused on the molecular mechanisms of TCMs to treat different diseases, for example, Shuang Kou et al. [5] reported that Zuo-Gui and You-Gui pills exerted neuroprotective effects by downregulation of NogoA, $\mathrm{NgR}$, and RhoA pathways in rats with experimental autoimmune encephalomyelitis. Some effective TCM formulas have also been found to treat SSc through acting on certain molecular targets. Yan et al. [6] reported that Wenyang Chubi Decoction had the effects in decreasing the connective tissue growth factor and collagen-I expression and improving the skin fibrosis in a mouse model of SSc. Yiqihuoxue formula was found to effectively reduce collagen production via downregulating the phosphorylation of Smad3 and then the activity of Smad binding element, which are involved in the TGF-beta pathway and constitutively activated in the progression of SSc [7].

Wenyang Huazhuo Tongluo (WYHZTL) is a patented formula (No. CN201310351880.2) to treat SSc owned by our team, and has shown the efficacy of anti-fibrosis in clinical applications. Our previous studies proved that it exerts its therapeutic effect on SSc patients by regulating Th17/Treg imbalance, lowering levels of von Willebrand factor (vWF) and aminoterminal propeptide of type III procollagen (PIIINP), and elevating the level of cross-linked carboxyterminal telopeptide of type I collagen (I CTP) [8]. We also reported that the formula antagonizes the fibrosis in a mouse SSc model through its regulation of TGF- $\beta 1$ /Smad signal pathway and the reduction of collagen content [9]. We also have demonstrated that the sera containing WYHZTL reduces the expression levels of collagen I/III through regulating some key signal molecules, such as TGF- $\beta 1$ receptor I, p-Smad2/3, Smad7, in TGF $\beta 1 /$ Smad Signaling pathway of skin fibroblasts obtained from SSc patients [10]. In another report, we found the sera containing WYHZTL inhibits the proliferation of SSc skin fibroblasts via blocking the cell cycle transition from the $\mathrm{G} 1$ to $\mathrm{S}$ phase [11], but the exact mechanism is still unclear. Therefore, further study on the role of WYHZTL in fibrosis of SSc is needed. It's known that the cell cycle transition from the G1 to $\mathrm{S}$ phase is regulated by cyclin D1, and cyclin D1 activation promotes the passage of cells through the G1 restriction checkpoint $[12,13]$. Apoptosis is a key mechanism involved in fibrosis of SSc [14], and survivin is an inhibitor of apoptotic protein gene family and a cell cycle regulator, it is critically required for suppression of apoptosis and ensuring normal cell division in the G2/M phase of the cell cycle [15].

In order to address the anti-fibrotic mechanism of WYHZTL formula in treating SSc, a novel seropharmacology method arising recent years for pharmacological study is taken to study TCM in vitro using animalmedicated sera [16]. The essence of seropharmacology is to administrate TCM to experimental animals (generally are rabbits or rats), followed by harvesting animal sera after a period of time. Then the sera are applied to different cell lines for molecular mechanism study. The advantage of seropharmacology for TCM study is to use the real pharmaceutical ingredients or internal biological metabolic compounds of TCM, without isolating the individual components, which is neither difficulty nor necessary in some cases.

The aim of the present study is to investigate the mechanism of WYHZTL formula against fibrosis in SSc regarding its effect on the expression and regulation of cyclin D1 and survivin in primary cultured human fibroblasts treated by the animal-mediated sera.

\section{Methods \\ Composition and preparation of Wenyang Huazhuo Tongluo formula}

The ingredients of WYHZTL formula were purchased from the First Affiliated Hospital of Nanyang Institute of Technology and the full ingredients are Radix Astragali membranacei, Herba Epimedii, Ramulus Cinnamomi cassiae, Herba Glechomae longitubae, Semen Sinapis albae, Radix Dioscoreae oppositae, Radix Codonopsitis pilosulae, Radix Rehmanniae praeparata, Fasciculus vascularis Luffae and Capparis zeylanica Linn. All of the ingredients of WYHZTL were prepared as crude slices 
and boiled twice (90 min per time) with ultrapure water as the doctor's directions. The water extracts were combined, filtered and evaporated under reduced pressure to a final concentration of $1.5 \mathrm{~g} / \mathrm{mL}$ based on the equivalent amount of the crude drugs.

\section{Cell culture}

Skin biopsy specimens were obtained from five progressive SSc patients, who had the disease course of less than 2 years and were fulfilled the American College of Rheumatology criteria for SSc [17]. Biopsy specimens $(3 \mathrm{~mm} \times 3 \mathrm{~mm}$ ) were performed at the edge of the lesion of each SSc patient. All patients provided written contents, and the study was approved by the Ethics Committee of Nanyang Institute of Technology, China.

Skin samples were transported in DMEM supplemented with $10 \%$ fetal bovine serum (FBS), $100 \mathrm{IU} / \mathrm{mL}$ penicillin, and $100 \mathrm{mg} / \mathrm{mL}$ streptomycin for processing the same day. The skin samples were washed in $75 \%$ ethanol, phosphate buffered saline (PBS), and the DMEM supplement. Cultured fibroblasts were established by mincing tissues and placing them into $60-\mathrm{mm}$ culture dishes secured by glass coverslips. Third-to sixth-passage fibroblasts were used for gene and protein expression assays.

\section{Preparation of rat medicated sera}

The rat medicated sera was prepared according to the published protocols [18]. Briefly, 30 Wistar female rats, aged between 6 and 8 weeks old and weighing $220 \sim$ $250 \mathrm{~g}$, were divided into WYHZTL $(N=15)$ and control $(N=15)$ groups. The animals were supplied by the Henan Experimental Animal Center (Zhengzhou, China) and maintained in accordance with accepted standards of humane animal care as outlined in ethical guidelines for care and use of laboratory animals in an air-conditioned room with controlled a temperature of $22 \pm 2{ }^{\circ} \mathrm{C}$, a humidity level of $45 \%$ to $65 \%$, and a $12 / 12 \mathrm{~h}$ light/dark cycle. The rats in WYHZTL group were individually administrated by gavage with WYHZTL decoction of $6.25 \mathrm{~g} / \mathrm{kg} /$ day based on clinical dosage. The control group received deionized water. After 10 days of administration, blood was collected from retinal venous plexus and centrifuged. The collected sera were aliquoted into $5 \mathrm{~mL}$ Eppendorf tubes and preserved at $-80{ }^{\circ} \mathrm{C}$ for future use.

\section{Sera samples preparation for HPLC analysis}

One milliliter of the rat medicated sera were mixed with $5 \mathrm{~mL}$ of methanol and ultrasonicated for $20 \mathrm{~min}$. The mixture was then centrifuged at $3000 \mathrm{rpm}$ for $10 \mathrm{~min}$ at room temperature, and the supernatant was evaporated to dryness under a steam of nitrogen at $50{ }^{\circ} \mathrm{C}$. The residues were reconstituted in $100 \mu \mathrm{L}$ of methanol and filtrated through a $0.45 \mu \mathrm{m}$ membrane filter (Millipore Co. Ltd., Tokyo, Japan), and an aliquot $(20 \mu \mathrm{L})$ was applied to the HPLC analysis. The HPLC analysis was performed with a Waters Alliance 2695 HPLC system. The HPLC conditions were as follows: column: Agilent C18 column $(150 \mathrm{~mm} \times 4.6 \mathrm{~mm})$; column temperature: $30{ }^{\circ} \mathrm{C}$; mobile phases: $0.1 \%$ ammonium acetate (A) and linear gradient system of methanol (B), where A/B: 85/15 (0 min), 70/30 (5 min), 30/70 (10 min), 30/70 (16 min), and $85 / 15$ (28 $\mathrm{min})$; flow rate: $1 \mathrm{~mL} / \mathrm{min}$; detection wavelength: $259 \mathrm{~nm}$; and injection volume: $20 \mu \mathrm{L}$.

\section{Proliferation assay}

Cell proliferation was analyzed with the cell counting kit-8 (CCK-8; Santa Cruz Biotechnology, USA) assay. Briefly, the fibroblast cells were harvested in exponential growth and seeded into 96 well plates at a density of $1.0 \times 10^{4}$ cells per well. The supernatant was removed after cell adhered to the wall and added FBS-free DMEM medium to incubate another $24 \mathrm{~h}$. And then the cells were treated with the rat medicated control sera, the WYHZTL sera or XAV939 (final concentration $2 \mathrm{ng} / \mathrm{mL}$; Sigma-Aldrich), a small-molecule inhibitor of tankyrase $1 / 2$ and $W n t / \beta$-catenin pathway, plus the WYHZTL sera respectively. The sera volume ratio for incubation is $15 \%$. After incubated 24,48 and $72 \mathrm{~h}$, CCK- 8 was added into the cells at a volume rate 1:10 to incubate for $2.5 \mathrm{~h}$. The absorbance was measured at $450 \mathrm{~nm}$ using an enzyme immunoassay analyzer (Dynatech MR4100, USA). The proliferation rate was calculated for each well. The mean and standard deviation of five relative proliferation rates for each well were calculated. The cell morphology was observed under an inverted microscope during the experimentation.

\section{Measurement of apoptosis by flow cytometry}

Analysis of apoptosis percentage was performed using an Annexin V-FITC/PI apoptosis kit (KeyGen Biotech, China) as previously described protocol [19]. Briefly, the cells were incubated with the rat medicated control sera, the WYHZTL sera and XAV939 plus the WYHZTL sera respectively for 24,48 and $72 \mathrm{~h}$. The sera volume ratio is same as above. When reaching $80 \%$ confluence, cells were trypsinized, washed with $\mathrm{PBS}$ and resuspended in $500 \mu \mathrm{l}$ of Annexin V-binding buffer. A mixture of $5 \mu \mathrm{l}$ of FITC-labeled Annexin V plus $5 \mu \mathrm{l}$ of Propidium Iodide (PI) solution was then added into the cells to incubate for $15 \mathrm{~min}$ at room temperature in the dark prior to flow cytometry analysis. Cell apoptosis was analyzed using CellQuest software (Becton-Dickinson). Three separate experiments were performed for each clone.

\section{Western blot analysis}

The effects of the rat medicated sera containing WYHZTL on cyclin D1 and survivin protein expression was analyzed as previously described protocol with slightly modification 
[20]. Briefly, the fibroblast cells were treated with the sera as above at a density of $1.0 \times 10^{9}$ cells per $25 \mathrm{ml}$ culture flask for 24, 48 and $72 \mathrm{~h}$, and then the cells were lysed using RIPA lysis buffer (Beyotime Institute of Biotechnology, China). The protein concentrations were determined using the bicinchoninic acid assay (BCA; Santa Cruz Biotechnology, USA). A total of $40 \mu \mathrm{g}$ of proteins was electrophoresed via sodium dodecyl sulphate polyacrylamide gel electrophoresis (SDS-PAGE), and transferred onto polyvinylidene fluoride (PVDF) membranes (Millipore, USA), which were then blocked with $5 \%$ skimmed milk powder for $1 \mathrm{~h}$ at room temperature. The membranes were incubated with antibodies against cyclin D1 and survivin (1: 1000 dilution; Santa Cruz Biotechnology, USA), followed by incubation with corresponding horseradish peroxidase (HRP)-conjugated secondary antibody at a 1:5000 dilution for $1 \mathrm{~h}$ at $37{ }^{\circ} \mathrm{C}$. Finally, the membranes were washed with PBS three times and the immunoreactive bands were visualized using an ECLPLUS/Kit according to the manufacturer's instructions. The optical density of each band was measured with a computer-assisted imaging analysis system (Quantity One, Bio-Rad, Hemel Hempstead, UK) and the relative protein levels were normalized to optical density of $\beta$-actin.

\section{RT-PCR analysis}

Total RNA from individual groups was extracted using the TRIzol reagent (Invitrogen, USA). All RNA preparation and handling steps took place in a laminar flow hood, under RNAse-free conditions. The isolated RNA from each fraction was dissolved in $20 \mu \mathrm{L}$ of RNAse-free water and stored at $-80{ }^{\circ} \mathrm{C}$ until used. cDNA synthesis was performed at $37{ }^{\circ} \mathrm{C}$ for $15 \mathrm{~min}$ and $85{ }^{\circ} \mathrm{C}$ for $5 \mathrm{sec}$ using the Primer Script RT reagent Kit (TaKaRa Biotechnology, Dalian, China) in a total volume of $20 \mu \mathrm{L}$ according to the manufacturer's instructions. The forward and reverse primers for amplifying cyclin D1 are as follows: $5^{\prime}$ CGATGCCAACCTCCTCAAC-3' and 5'-AAGCCTTGT AATCCTTGTG-3', and the length of the amplified fragment was $212 \mathrm{bp}$. The forward and reverse primers for amplifying survivin were as follows: 5'-GGACCACCG CATCTCTACA-3' and 5'-GCACTTTCTTCGCAGTTT CC-3', and the length of the amplified fragment was 338 bp. A 196-bp fragment of GAPDH was used as the internal control and was amplified with the following forward and reverse primers: 5'-GAGTCAACGGATTTG GTCGT-3' and 5'-GACAAGCTTCCCGTTCTCAG-3'. The PCR cycling conditions comprised a denaturation step for $5 \mathrm{~min}$ at $95^{\circ} \mathrm{C}$, followed by 30 cycles of denaturation (94 ${ }^{\circ} \mathrm{C}$ for $15 \mathrm{~s}$ ), annealing $\left(55^{\circ} \mathrm{C}\right.$ for cyclin $\mathrm{D} 1,54{ }^{\circ} \mathrm{C}$ for survivin and $57{ }^{\circ} \mathrm{C}$ for GAPDH for 30s), and extension (72 ${ }^{\circ} \mathrm{C}$ for $30 \mathrm{~s}$ ). After the last cycle, all PCR products were subjected to a final extension for $10 \mathrm{~min}$ at $72{ }^{\circ} \mathrm{C}$. PCR products were combined and then electrophoresed on
$1.5 \%$ agarose gels containing ethidium bromide; the images were captured using a Gel Doc XR+ Image Station (Bio-Rad, Hemel Hempstead, UK). The optical density of each band was measured with Quantity One and normalized to optical density of the housekeeping gene GAPDH.

\section{Statistical analysis}

Data were analyzed with SPSS 16.0. Statistical data are expressed as mean \pm SD. Group comparisons were performed using one-way ANOVA followed by a Tukey test for multiple comparisons. The results were considered statistically significant when $P<0.05$. Graphic representation was performed using GraphPad Prism version 6.01 for Windows (GraphPad Software, San Diego CA, USA, www.graphpad.com).

\section{Results}

Analysis of the compounds absorbed into the blood after oral administration of WYHZTL decoction in SD rats

Figure 1 shows the representative HPLC profiles of the control rat sera and the rat medicated sera, respectively. Four distinct peaks can be detected in the rat medicated sera containing WYHZTL compared with the control rat sera, which indicates that the rat medicated sera may contain active ingredients and/or metabolic components of the decoction.

\section{Effect of rat medicated sera containing WYHZTL on proliferation of fibroblasts}

In order to confirm the role of WYHZTL formula in the proliferation of fibroblasts, the rat medicated sera containing WYHZTL were added into the primary culture fibroblasts. The morphology of the cells was observed directly under an inverted microscope. In the control group, fibroblasts were numerous and having branched cytoplasm surrounding elliptical, speckled nucleus with two or more nucleoli. Most nucleoli were spindle and some were irregular, but all tended to be in the same direction. After incubated with XAV939 or the sera containing WYHZTL, both the total cell and the irregular cells with multi-nucleoli decreased, the cell bodies, nucleoli and the branched cytoplasm were shorter, compared with those in the control group (as shown in Fig. 2a). Such results demonstrated that the sera containing WYHZTL strongly implicated in cell morphology regulation and had inhibitory effect on proliferation in fibroblasts.

The above evidence was further confirmed by CCK- 8 assay. After incubated with the rat medicated sera or XAV939 for 28, 48 and $72 \mathrm{~h}$, the proliferative ability of the fibroblasts cells was markedly reduced and was significantly lower than that in the control group following the indicated time of incubation $(P<0.01)$, as shown in Fig. 2b. 

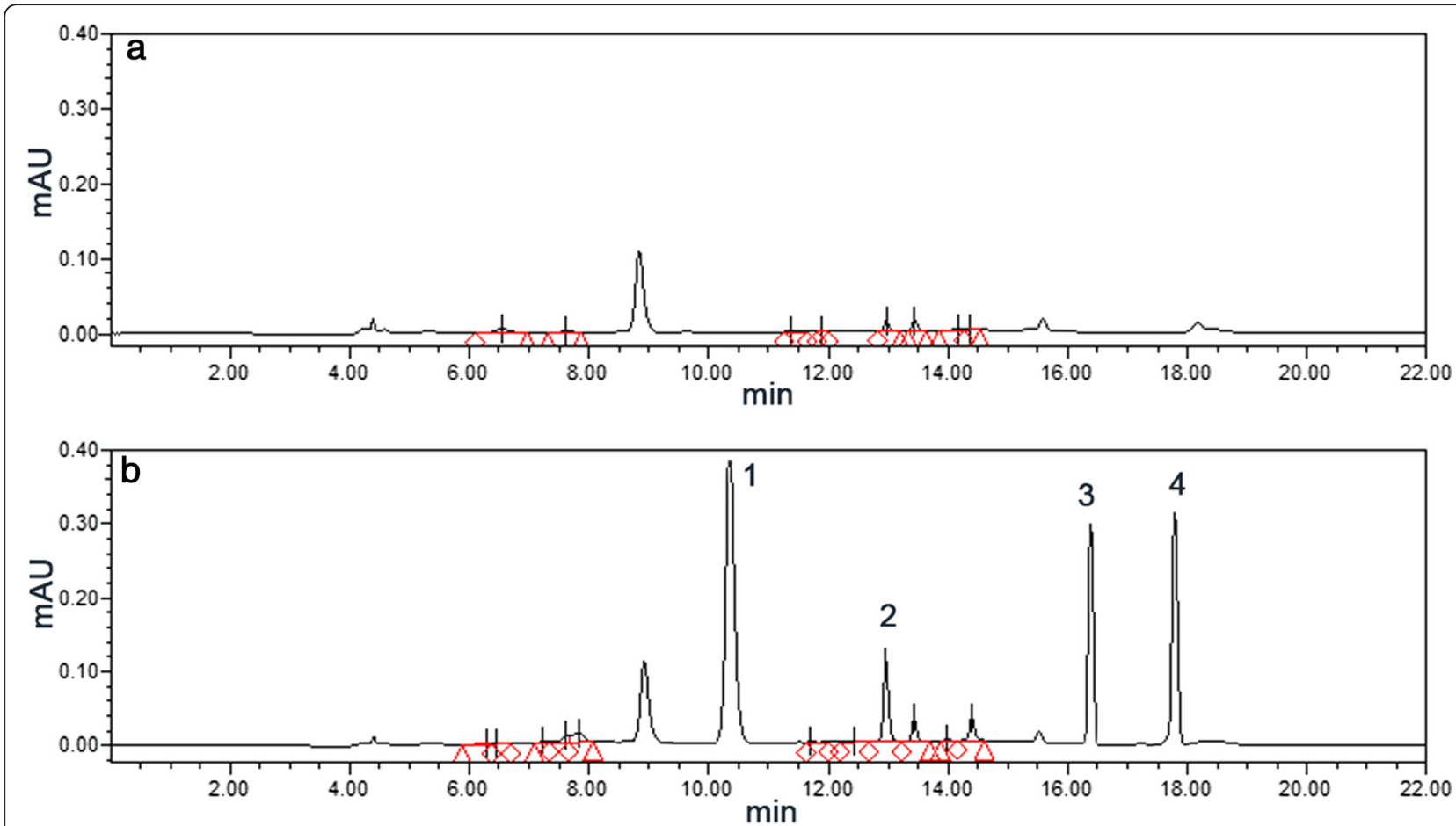

Fig. 1 HPLC profiles of the rat medicated sera after administrated by gavage of the WYHZTL decoction. a Control rat sera; $\mathbf{b}$ rat medicated sera after administrated by gavage $1 \mathrm{~h}$. Four distinctive peaks (1, 2, 3 and 4$)$ are detected in the rat medicated sera, but not in the control rat sera. This indicates that the rat medicated sera may contain active ingredients and/or metabolic components of the WYHZTL decoction

a

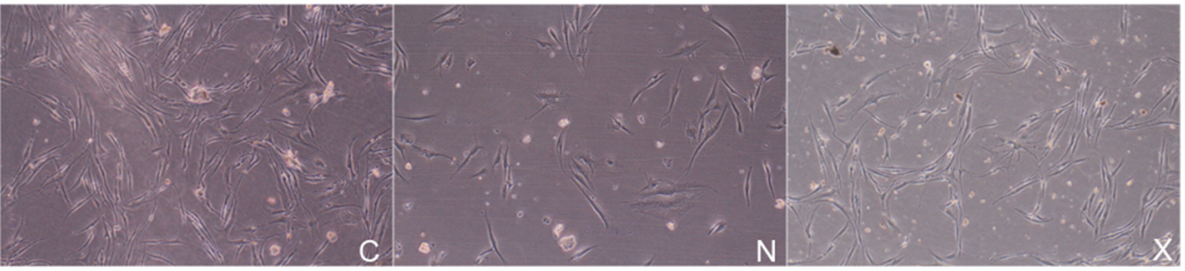

b

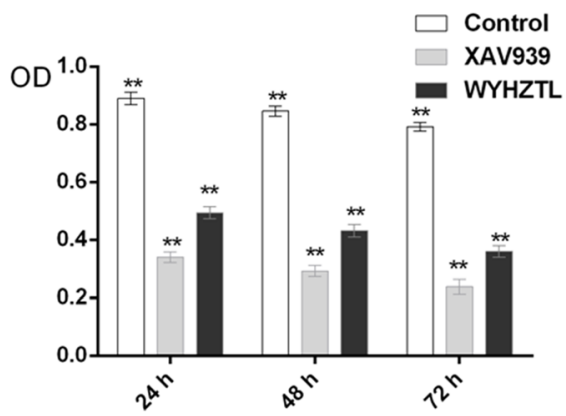

Fig. 2 Effect of Rat Medicated Sera containing WYHZTL on proliferation of fibroblasts. a Cell morphology observation: cells cultured in DMEM containing the sera were grown in the flask and viewed directly under the Nikon Phase Contrast Inverted Microscopy (200x original magnification; Ti-S type, Nikon Co., Japan). b The CCK-8 assay was performed to examine cell proliferation. The absorbance measured at $450 \mathrm{~nm}$ by using an enzyme immunoassay analyzer (Dynatech MR4100, USA) after incubated for 24, 48 and $72 \mathrm{~h}$. The mean and standard deviation of 5 relative proliferation rates for each well were calculated. C represents control group, N represents XAV939 inhibitor group, X represents rat medicated sera containing WYHZTL group. ${ }^{*} P<0.01$ compared with each group 


\section{Rat medicated sera containing WYHZTL promotes apoptosis in fibroblasts}

In order to determine the effects of the rat medicated sera containing WYHZTL on apoptosis in fibroblasts, viable, early apoptotic, and late apoptotic or necrotic cells can be distinguished by flow cytometry analysis using dual staining with Annexin V/PI dyes. As shown in Fig. 3a, viable cells were PI and Annexin V-FITC double negative; apoptotic cells were Annexin V-FITC positive and PI negative, whereas late apoptotic or necrotic cells were Annexin V-FITC and PI double positive. We observed a strong time-dependent relationship with regard to the rat medicated sera containing WYHZTL or XAV939 exposure and early apoptotic or late apoptotic cells (Fig. 3b, c). The percentage of late apoptotic cell population in the sera containing WYHZTL treated cells was clearly higher than that in those treated with the control sera and was slightly lower than that in those treated with XAV939 $(P<0.01)$. Interestingly, the percentage of early apoptotic cell population in the sera containing WYHZTL treated cells was clearly higher than that in those treated with XAV939 $(P<0.01)$, which suggesting the rat medicated sera containing WYHZTL promotes apoptosis, especially early apoptosis, in the fibroblasts. This effect was also observed in XAV939 treated cells.
Effect of rat medicated sera containing WYHZTL on cyclin D1 in fibroblasts

In order to further explore the molecular mechanisms of the role of the rat medicated sera containing WYHZTL, we focused on the recognized proliferation-associated gene cyclin D1. RT-PCR and Western blotting were performed to determine the cyclin D1 mRNA and protein levels after treated with the rat medicated sera containing WYHZTL in fibroblasts. As shown in Fig. 4, treated with the rat medicated sera containing WYHZTL for 24, 48 and $72 \mathrm{~h}$ led to a down-regulation of cyclin D1 mRNA and protein expression compared with that in the control group $(P<0.01)$, in accordance with the effect of XAV939 known to have inhibition action on cyclin D1 $(P>0.05)$. These results indicated that the sera inhibited proliferation in fibroblasts through downregulation of cyclin D1 in mRNA and protein level.

\section{Effect of rat medicated sera containing WYHZTL on survivin in fibroblasts}

The role of the rat medicated sera containing WYHZTL on the recognized apoptosis-associated gene survivin was examined by RT-PCR and Western blotting in fibroblasts. As shown in Fig. 5, treating with the rat medicated sera containing WYHZTL led to a downregulation of survivin mRNA and protein expression compared to the control group $(P<0.01)$. The sera had
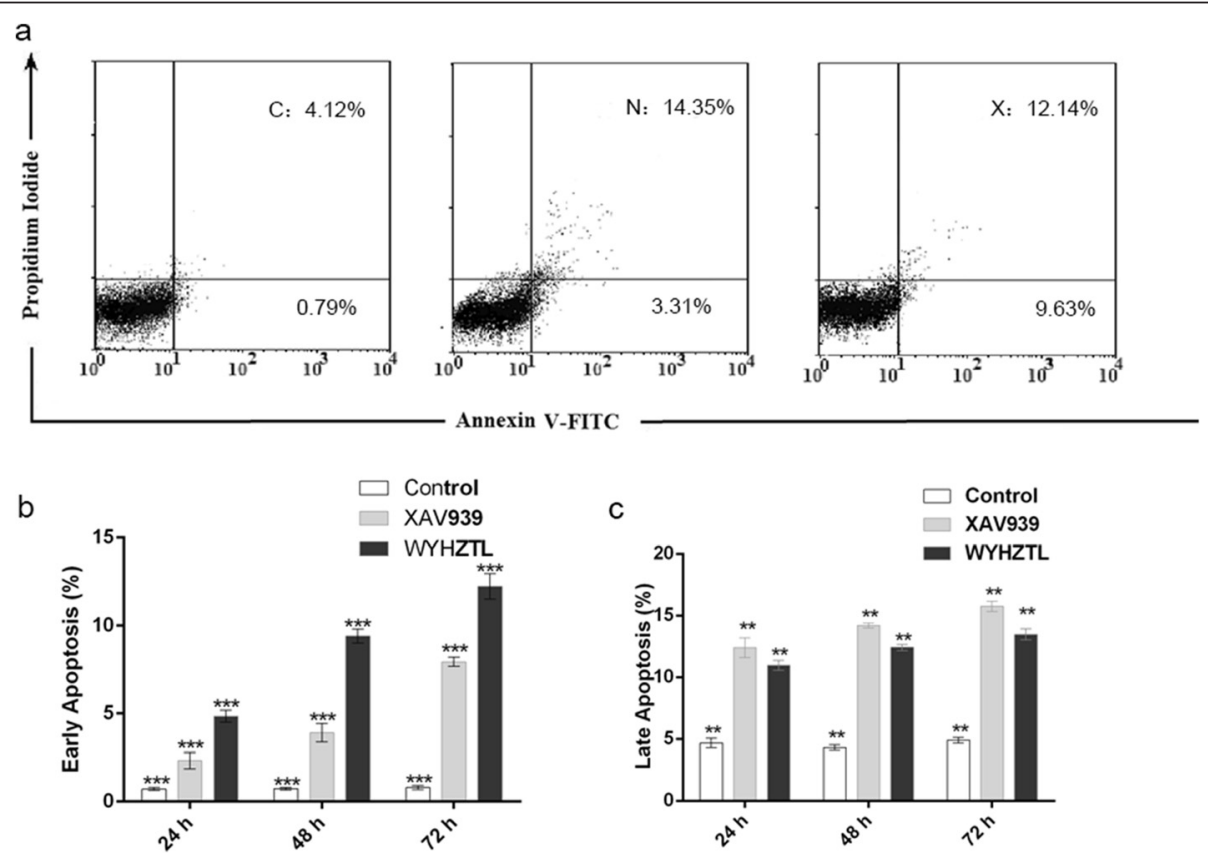

Fig. 3 Rat medicated sera containing WYHZTL induces cell apoptosis in fibroblasts. a Cell apoptosis was examined after incubation for 48 h by Annexin V-PI staining using flow cytometry. Representative results are shown. $\mathbf{b}$ Bar graph of early apoptosis rate after incubated with the sera or XAV939 for 24, 48 and 72 h. c Bar graph of late apoptosis rate after incubated with the sera or XAV939 for 24, 48 and 72 h. C represents control group, $\mathrm{N}$ represents XAV939 inhibitor group, $X$ represents rat medicated sera containing WYHZTL group. ${ }^{* *} P<0.01$, ${ }^{* * *} P<0.001$ compared with each group. The results are the mean \pm SD of 3 independent experiments 

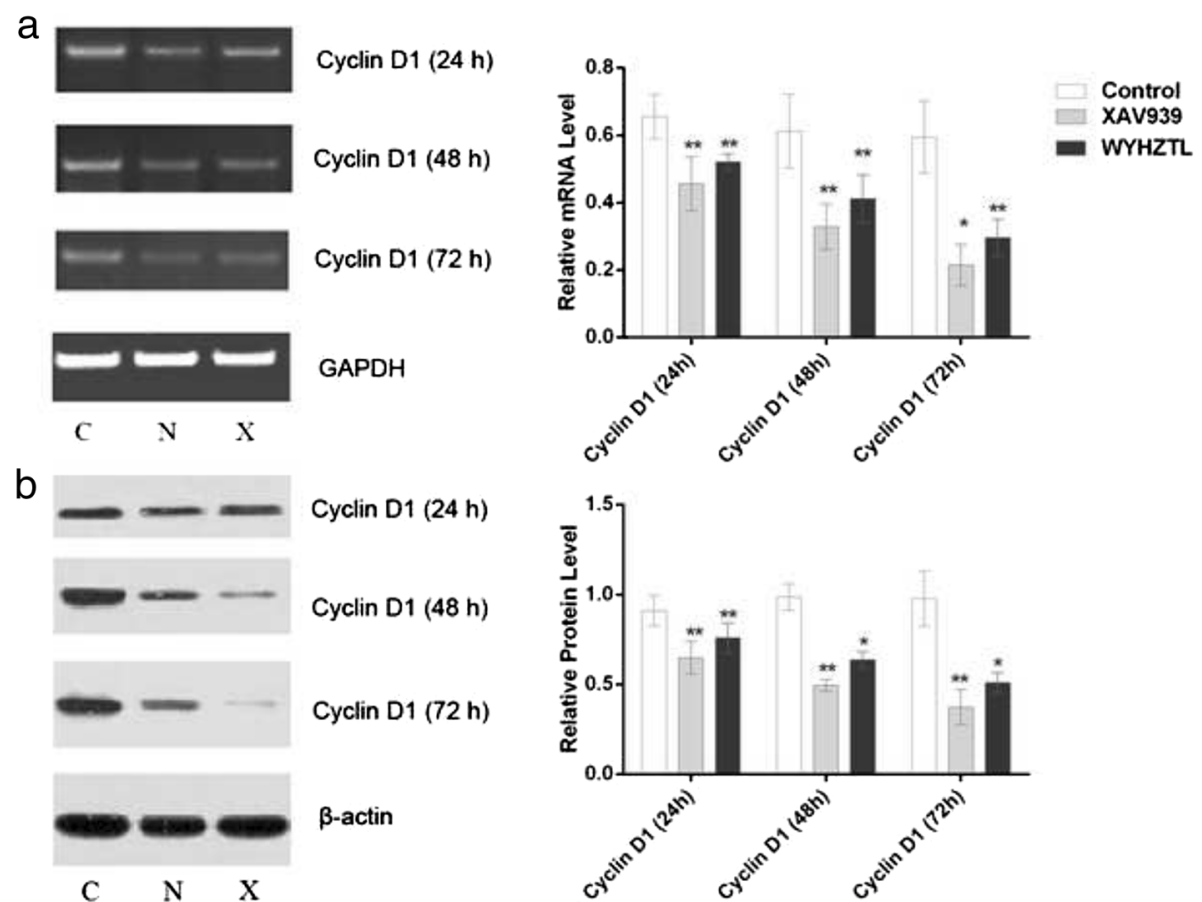

Fig. 4 Effect of down-regulation of cyclin D1 by the rat medicated sera containing WYHZTL in fibroblasts. a The mRNA levels of cyclin D1 were detected by RT-PCR after treated with the sera containing WYHZTL for 24, 48 and 72 h. GAPDH was applied as an endogenous control and densitometric values were normalized by GAPDH. b The protein levels of cyclin D1 were detected by Western blotting after treated with the sera containing WYHZTL for 24,48 and 72 h. $\beta$-actin was used as an endogenous control and densitometric values were normalized by $\beta$-actin. Representative results are shown. C represents control group, N represents XAV939 inhibitor group, X represents Rat Medicated Sera containing WYHZTL group. Densitometric values are shown as mean \pm SD. Left panels represent RT-PCR/Western blotting gel results, right panels represent bar graphs for relative mRNA and protein levels for cyclin D1. ${ }^{*} P<0.05,{ }^{* *} P<0.01$, compared with the control group. The results are the mean \pm SD of at least 5 independent experiments

a similar inhibitory effect on fibroblasts to XAV939 although the former is weaker than the later $(P>0.05)$. These results indicated that the sera promoted apoptosis in fibroblasts via down-regulation of survivin in both mRNA and protein levels.

\section{Discussion}

SSc is a chronic fibrosing connective tissue disease characterized by a higher mortality rate compared with other connective tissue diseases. In early stages of SSc, the main pathological manifestations are perivascular inflammatory infiltrates and a reduced capillary density, whereas later stages are fibrosis characterized by an excessive accumulation of collagen-rich ECM. The fibrosis disrupts the physiological structure of the affected tissues and interferes with proper organ function. Tissue fibrosis is the primary cause of SSc-related mortality presently and the 10 -year survival rate is as low as $54 \%$ - $66 \%$ [21]. There is a high unmet clinical need for effective antifibrotic therapies in SSc, but no drugs have been clearly shown to be effective in reducing specifically the development of SSc fibrosis in clinic up to now $[4,22]$.

Chinese medical treatment is aimed at adjusting the environmental and human influences through the use of
TCM, which exerts a comprehensive effect on the diseases; because the decoction is usually composed of several herbs or minerals according to the symptoms of patients. WYHZTL formula is composed of 10 herbs, among which Radix Astragali membranacei exerts an anti-fibrotic effect in rats induced by porcine serum via down-regulating PDGFR- $\beta$, inhibiting hepatic stellate cells proliferation and MAPK activation [23]. One of the isolated constituents from Herba Glechomae longitubae, asiatic acid, inhibits TGF- $\beta 1$-induced collagen and PAI- 1 expression in keloid fibroblasts through PPAR- $\gamma$ activation [24]; Radix Dioscoreae oppositae attenuats CCl (4)induced hepatic fibrosis in rats in a dose-dependent manner and the attenuation may be related to the antioxidant properties of Radix Dioscoreae oppositae [25]. The ethanolic extract of Capparis zeylanica Linn was reported having anti-fibrotic effect via inhibiting the fibroblast proliferation and type I collagen production in SSc [26]. However, the therapeutic action of a TCM formula is far more than that of the total sum of its components, it's a very complicated process in vivo. TCM formulas prescribed by doctors of Chinese medicine are selected on the basis of the past experience with treating diseases and on the basis of the current health 

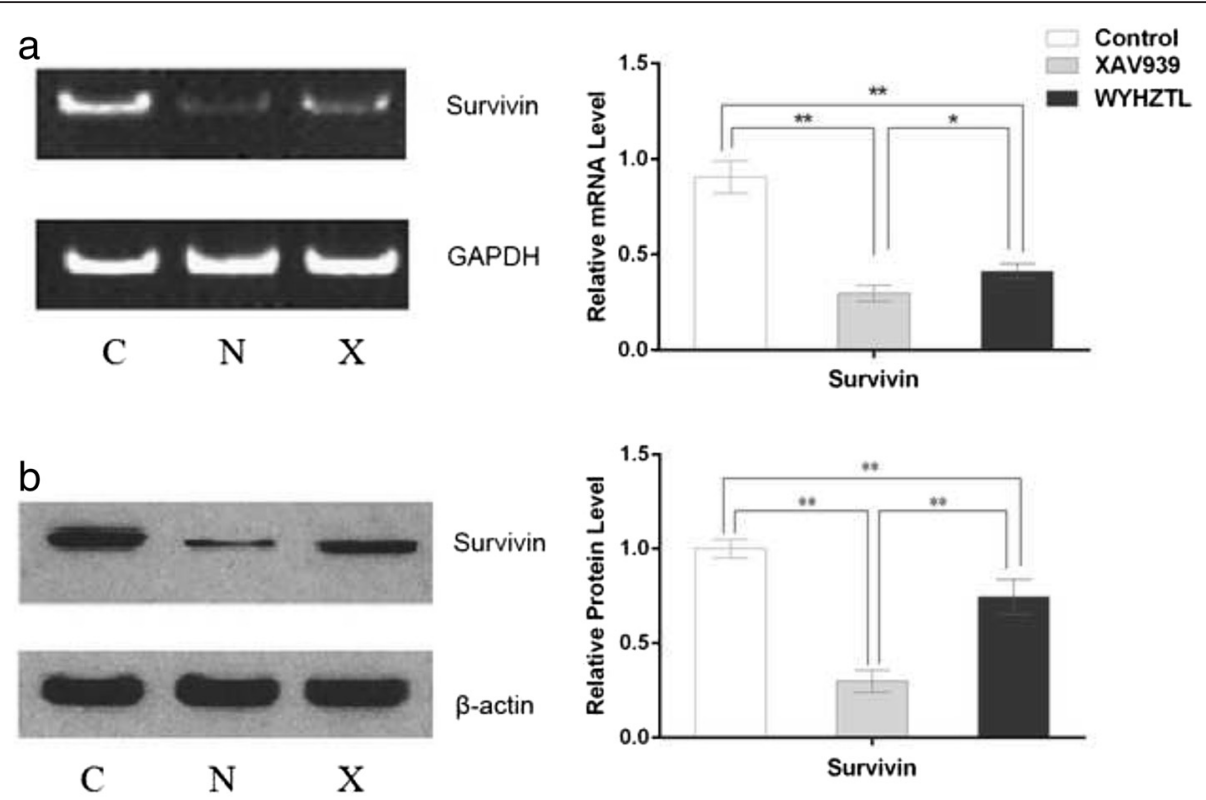

Fig. 5 Effect of down-regulation of survivin by the rat medicated sera containing WYHZTL in fibroblasts. a The mRNA levels of survivin were detected by RT-PCR after treated with the sera containing WYHZTL for $48 \mathrm{~h}$. GAPDH was applied as an endogenous control and densitometric values were normalized by GAPDH. $\mathbf{b}$ The protein levels of survivin were detected by western blotting after treated with the sera containing WYHZTL for $48 \mathrm{~h}$. $\beta$-actin was used as an endogenous control and densitometric values were normalized by $\beta$-actin. C represents control group, N represents XAV939 inhibitor group, $X$ represents rat medicated sera containing WYHZTL group. Representative results are shown. Densitometric values are shown as mean \pm SD. Left panels represent RT-PCR/Western blotting gel results, right panels represent bar graphs for relative mRNA and protein levels for survivin. ${ }^{*} P<0.05,{ }^{*} P<0.01$. The results are the mean \pm SD of at least 5 independent experiments

status of the individual patient. Not only do the symptoms of SSc vary from individual to individual, but also the health histories (such as coexisting diseases or syndromes) are different. These factors must be accounted in determining suitable prescriptions. Thus, TCM approach is a comprehensive treatment based on individual needs.

Known abnormalities in SSc that relate to the fibrotic response include fibroblast proliferation increased and apoptosis decreased [27]. So one of treatment strategies for fibrosis in SSc is to inhibit proliferation and promote apoptosis. Cyclins play an important role in cell progression of fibrosis, so the mRNA and protein levels of cell cycle regulatory factor, cyclin D1, were examined. Our results showed that incubation with WYHZTL formula in fibroblasts caused a marked reduction of the mRNA and protein levels of cyclin D1, indicating its crucial for the $\mathrm{G} 1$ to $\mathrm{S}$ transition $[12,13]$ and suppression of apoptosis [28]. Marsillach et al. reported that survivin expression is significantly increased during the development of fibrosis [29]. Consistently, Sisson et al. reported that inhibition of survivin restores susceptibility of fibroblasts to Fas-mediated apoptosis. Therefore, survivin may represent a potential target for anti-fibrotic therapies [30]. Additionally, survivin is critically required for suppression of apoptosis and ensuring normal cell division in the $\mathrm{G} 2 / \mathrm{M}$ phase of the cell cycle [15]. Our results showed that WYHZTL formula incubation in fibroblasts also caused significant reduction of the mRNA and protein levels of survivin. We have reported that WYHZTL formula has the effect of inhibition on proliferation of SSc skin fibroblasts via blocking the cell cycle transition from the G1 to S phase previously [11]. Therefore, our study suggested that WYHZTL formula arrested the cell cycle in the phase by inhibiting cyclin D1 expression, and its anti-fibrosis effect related to inhibition of cylcin D1 and survivin.

$\mathrm{XAV}-939$ is screened out as an inhibitor of $\mathrm{Wnt} / \beta$ catenin pathway and demonstrates to stabilize the axin levels through inhibiting tankyrases, and consequently inhibits the Wnt signaling and the expression of target genes, including survivin and cyclin D1 [31]. Aberrant $\mathrm{Wnt} / \beta$-catenin signaling pathway due to gain of $\beta$ catenin function, induces constitutive transcription of cyclin D1 $[28,32]$. Our results showed that the effect of WYHZTL formula on survivin and cyclin D1 is consistent with that of XAV939, this further proves that the anti-fibrosis effect of the formula is related to the inhibition of cylcin D1 and survivin.

\section{Conclusions}

In summary, our study demonstrates the WYHZTL formula has antiproliferative and pro-apoptotic actions on fibroblasts in SSc. It also provides valuable information 
regarding the mechanism of repression of SSc fibrosis, and the effect may be related to the down-regulation of mRNA and protein levels of cyclin D1 and survivin in SSc. Nevertheless, further studies on the other possible mechanisms of anti-fibrosis effect of WYHZTL formula and on the actual active ingredients and/or biotransformed ingredients contributed to the anti-fibrosis effect of WYHZTL are needed.

\begin{abstract}
Abbreviations
ECM: excessive accumulation of extracellular matrix; I CTP: cross-linked carboxyterminal telopeptide of type I collagen; MAPK: mitogen-activated protein kinase; PAI-1: plasminogen activator inhibitor-1; PDGFR- $\beta$ : plateletderived growth factor receptor- $\beta$; PIIINP: aminoterminal propeptide of type III procollagen; PPAR- : peroxisome proliferator-activated receptor- ; SSc: systemic sclerosis; TCM: traditional Chinese medicine; TGF- $\beta$ : transforming growth factor beta; vWF: von Willebrand factor; WYHZTL: Wenyang Huazhuo Tongluo formula.
\end{abstract}

\section{Competing interests}

The authors declare that the research was conducted in the absence of any commercial or financial relationships that could be construed as a potential conflict of interest. Dr. Hua Bian holds a patent on the tested WYHZTL formula (No. CN201310351880.2)

\section{Authors' contributions}

LH carried out the molecular studies and drafted the manuscript. HB provided the WYHZTL formula, designed the study and performed the statistical analysis. JO carried and conceived of the study, YB participated in the study design and helped to draft the manuscript. LY prepared with the rat sera. SY helped the in vitro study. All authors read and approved the final manuscript.

\section{Acknowledgements}

The present study was supported by the National Natural Science Foundation of China (grant NO. 81373627).

\section{Author details}

'Zhang Zhongjing College of Chinese Medicine, Nanyang Institute of Technology, Changjiang Road 80, Nanyang 473004Henan, China. 2Experimental Research Center, China Academy of Chinese Medical Sciences, Beijing, China. ${ }^{3}$ School of Pharmaceutical Sciences, Zhengzhou University, Zhengzhou, Henan, China.

Received: 17 September 2015 Accepted: 16 February 2016 Published online: 20 February 2016

\section{References}

1. Lafyatis R. Transforming growth factor beta-at the centre of systemic sclerosis. Nat Rev Rheumatol. 2014; doi:10.1038/nrrheum.2014.137.

2. Bagnato GL, Roberts WN, Fiorenza A, et al. Skin fibrosis correlates with circulating thyrotropin levels in systemic sclerosis: translational association with Hashimoto's thyroiditis. Endocrine. 2015; doi:10.1007/s12020-015-0600-3.

3. Antic M, Distler JH, Distler O. Treating skin and lung fibrosis in systemic sclerosis: a future filled with promise. Curr Opin Pharmacol. 2013;13(3):455-62.

4. Jordan S, Chung J, Distler O. Preclinical and translational research to discover potentially effective antifibrotic therapies in systemic sclerosis. Curr Opin Rheumatol. 2013;25(6):679-85.

5. Kou S, Zheng Q, Wang Y, et al. Zuo-Gui and You-Gui pills, two traditional Chinese herbal formulas, downregulated the expression of NogoA, NgR, and RhoA in rats with experimental autoimmune encephalomyelitis. J Ethnopharmacol. 2014;158:102-12.

6. Yan XN, Feng J, Li WB, Cui R, Shi BJ. Effects of Wenyang Chubi Decoction on connective tissue growth factor and collagen-I in a mouse model of scleroderma. Zhong Xi Yi Jie He Xue Bao. 2007;5(5):526-30.

7. Wu T, Chu H, Tu W, et al. Dissection of the mechanism of traditional Chinese medical prescription-Yiqihuoxue formula as an effective anti-fibrotic treatment for systemic sclerosis. BMC Complement Altern Med. 2014;14:224

8. Hua B, Min Y, Gao Z, et al. Effect of Wenyang Huazhuo Tongluo Recipe on Peripheral Blood Th17/Treg Cell Balance In Systemic Sclerosis Patient. Zhongguo Zhongxiyi Jiehe Zazhi. 2015;35(08):975-9.
9. Bian $H$, Lv Q, Han L, et al. Impact of Wenyang Huazhuo Tongluo Formula on TGF- $\beta 1$ /Smad Signal Pathway of Systemic Sclerosis Mouse Model. J Tradit Chin Med. 2015;56(04):327-31.

10. Bian H, Qin LV, Huang $X$, et al. The effects of Wenyang Huazhuo Tongluo Recipe On TGF- $31 /$ Smad Signaling pathway of fibroblasts in Systemic sclerosis patients. Zhongguo Zhongxiyi Jiehe Zazhi. 2015;35(9):1054-8.

11. Bian H, Fan YS, Lou LH, Mao BY, Shi JY. Effect of Wenyang Huazhuo Tongluo recipe contained serum on proliferation and cell cycle of systemic sclerosis skin fibroblasts. Zhong Yao Cai. 2009;32(6):936-9.

12. Blagosklonny MV, Pardee AB. The restriction point of the cell cycle. Cell Cycle. 2002;1(2):103-10.

13. Moroy T, Geisen C. Cyclin E. Int J Biochem Cell Biol. 2004;36(8):1424-39.

14. Chabaud S, Moulin VJ. Apoptosis modulation as a promising target for treatment of systemic sclerosis. Int J Rheumatol. 2011;2011:495792.

15. Cheung $\mathrm{CH}$, Chen $\mathrm{HH}$, Kuo CC, et al. Survivin counteracts the therapeutic effect of microtubule de-stabilizers by stabilizing tubulin polymers. Mol Cancer. 2009:8:43.

16. Xu H, Wu Q, Peng C, Zhou L. Study on the antiviral activity of San Huang Yi Gan Capsule against hepatitis B virus with seropharmacological method. BMC Complement Altern Med. 2013:13:239.

17. van den Hoogen F, Khanna D, Fransen J, et al. 2013 classification criteria for systemic sclerosis: an American college of rheumatology/European league against rheumatism collaborative initiative. Ann Rheum Dis. 2013;72(11):1747-55.

18. Hu X, Lu H, Deng YL, Wan Q Yie SM. Effect of Rat Medicated Serum Containing Zuo Gui Wan and/or You Gui Wan on the Differentiation of Stem Cells Derived from Human First Trimester Umbilical Cord into Oocyte-Like Cells In Vitro. Evid Based Complement Alternat Med. 2015;2015:825805.

19. Wang $P$, Yin B, Shan $L$, et al. RNA interference-mediated knockdown of astrocyte elevated gene-1 inhibits growth, induces apoptosis, and increases the chemosensitivity to 5-Fluorouracil in renal cancer caki-1 cells. Mol Cells. 2014:37(12):857-64

20. Han L, Wang Y, Guo X, et al. Downregulation of MDR1 Gene by Cepharanthine Hydrochloride Is Related to the Activation of c-Jun/JNK in K562/ADR Cells. Biomed Res Int. 2014;2014:164391

21. Steen VD, Medsger TA. Changes in causes of death in systemic sclerosis, 1972-2002. Ann Rheum Dis. 2007;66(7):940-4.

22. Beyer C, Distler $\mathrm{O}$, Distler JH. Innovative antifibrotic therapies in systemic sclerosis. Curr Opin Rheumatol. 2012;24(3):274-80.

23. Sun WY, Wang L, Liu H, Li X, Wei W. A standardized extract from Paeonia lactiflora and Astragalus membranaceus attenuates liver fibrosis induced by porcine serum in rats. Int J Mol Med. 2012;29(3):491-8.

24. Bian D, Zhang J, Wu X et al. Asiatic acid isolated from Centella asiatica inhibits TGF-beta1-induced collagen expression in human keloid fibroblasts via PPAR-gamma activation. Int J Biol Sci. 2013;9(10):1032-42.

25. Chan YC, Chang SC, Liu SY, Yang HL, Hseu YC, Liao JW. Beneficial effects of yam on carbon tetrachloride-induced hepatic fibrosis in rats. J Sci Food Agric. 2010;90(1):161-7.

26. Cao YL, Li X, Zheng M. Effect of Capparis spinosa on fibroblast proliferation and type I collagen production in progressive systemic sclerosis. Zhongguo Zhong Yao Za Zhi. 2008:33(5):560-3.

27. Kissin EY, Korn JH. Fibrosis in scleroderma. Rheum Dis Clin North Am. 2003; 29(2):351-69.

28. Choi YJ, Anders L. Signaling through cyclin D-dependent kinases. Oncogene. 2014;33(15):1890-903.

29. Marsillach J, Ferre N, Camps J, Rull A, Beltran R, Joven J. Changes in the expression of genes related to apoptosis and fibrosis pathways in $\mathrm{CCl} 4$ treated rats. Mol Cell Biochem. 2008:308(1-2):101-9.

30. Sisson TH, Maher TM, Ajayi IO, et al. Increased survivin expression contributes to apoptosis-resistance in IPF fibroblasts. Adv Biosci Biotechnol. 2012;3(6A):657-64.

31. Esfandiari F, Fathi A, Gourabi H, Kiani S, Nemati S, Baharvand H. Glycogen synthase kinase-3 inhibition promotes proliferation and neuronal differentiation of human-induced pluripotent stem cell-derived neural progenitors. Stem Cells Dev. 2012;21(17):3233-43.

32. Shtutman M, Zhurinsky J, Simcha I, et al. The cyclin D1 gene is a target of the beta-catenin/LEF-1 pathway. Proc Natl Acad Sci U S A. 1999:96(10):5522-7. 